\title{
Estudo de um modelo bidimensional de reator de plasma RF com acoplamento capacitivo simulado pelo método de elementos finitos
}

\section{Study of a bidimensional model of RF plasma reactor with capacitive coupling simulated by the finite elements method}

Carla Andreia dos Santos Melo1,* (D), Marcelo Bento Pisani', Gesil Sampaio Amarante Segundo ${ }^{1}$ (D)

1. Universidade Estadual de Santa Cruz - Ilhéus (BA), Brasil

Autor correspondente: melo.carla2011@gmail.com

Editor de seção: Maria Lúcia P Silva

Recebido: Dez. 13, 2020 Aceito: Fev. 15, 2021

\begin{abstract}
RESUMO
Neste trabalho estudamos um plasma de acoplamento capacitivo com fonte com radiofrequência de 13,56 MHz e pressão a 13,33 Pa utilizando o código finite elements method (FEM) para a simulação de um modelo bidimensional de descarga de argônio. É de extrema importância conhecer o que ocorre na câmara de deposição de filmes finos na expectativa de alcançar a redução de custos na produção de filmes finos. Os resultados demonstram, considerando o comprimento de Debye e pela evidência da presença de bainhas, o efeito de blindagem no plasma. A densidade de elétrons encontrada está em concordância com o modelo global aproximado de descarga descrito previamente. Variações de parâmetros como a potência fornecida, na faixa de 1 a $100 \mathrm{~W}$, gerou um plasma mais energético com a densidade de elétrons entre $1,5 \times 10^{15}$ e $5 \times 10^{16} \mathrm{~m}^{-3}$. No modelo foi possível visualizar na distribuição espacial do plasma a formação e a assimetria da bainha, o que possibilitou estabelecer a posição de maior densidade nos eixos axial e radial. Adicionalmente, essas distribuições demonstram regiões não uniformes de densidade, o que é confirmado pela observação experimental de uma maior erosão do alvo, e os filmes depositados apresentam espessuras menores das bordas axiais do substrato.
\end{abstract}

PALAVRAS-CHAVE: Plasma, Modelagem e simulação, Densidade dos elétrons.

\begin{abstract}
In this work, we have studied a capacitive coupling plasma with source at 13.56-MHz radiofrequency and pressure at $13.33 \mathrm{~Pa}$ using finite elements method (FEM) for the simulation of two-dimensional model. It is extremely important to know what happens in the deposition chamber of thin films seeking results that provide the production of thin films with less material resources. By the Debye length and the presence of sheath, the results demonstrated the plasma shielding effect. A parametric study conducted by the supplied power ranging from 1 to $100 \mathrm{~W}$ generated a more energetic plasma, and the initially obtained electron density of $1.5 \times 10^{15} \mathrm{~m}^{-3}$ increased to $5 \times 10^{16} \mathrm{~m}^{-3}$. In the model, we could visualize the spatial distribution of the plasma, which demonstrates the formation and asymmetry of the sheath and made it possible to establish the position in which it reaches the highest density in the axial and radial axes. Additionally, the density distribution confirmed the predisposition of the deposited films to have a smaller thickness measurement on the axial edges of the substrate and the erosion of the target, resulting from successive depositions, what is related to regions in which the plasma density is higher, presenting higher target consumption.
\end{abstract}

KEYWORDS: Plasma, Modelling and Simulation, Electron density. 


\section{INTRODUÇÃO}

Os filmes finos podem ser obtidos por uma variedade de técnicas disponíveis, tais como a deposição por evaporação, a deposição física ou química a partir da fase vapor e a corrosão por bombardeio iônico. A pulverização por radiofrequência (RF) ou sputtering RF é considerada uma importante técnica de deposição'. A deposição ocorre em uma superfície (substrato) pelo bombardeamento por íons (gás inerte) por meio da aplicação de alta energia (plasma). O material ejetado do alvo é depositado em todas as direções no substrato formando camadas do material selecionado presente no alvo.

Um método disponível para o estudo de técnicas de deposição é o desenvolvimento de modelos matemáticos. Podem-se obter, interpretar e validar resultados advindos de um modelo de um sistema físico em estudo. O uso do método de elementos finitos, que tem disponibilidade de modelo de plasma frio adequado para a simulação de ambientes de deposição assistida a plasma desde 2010, possui poucos estudos publicados, sendo o método particle in cell/Monte Carlo collision, ou partícula em célula/colisões modeladas pelo método Monte Carlo (PIC/MCC), o mais reconhecido, com a precisão de seus resultados já comprovada por vários estudos independentes².

Nesse contexto, o modelo busca compreender o que ocorre dentro da câmara de deposição da evaporadora Prest Vácuo 600, que é um sistema constituído de uma câmara de aço inoxidável, um conjunto de bombas de vácuo, controladores e injetores de gases, medidor de pressão, uma fonte de tensão de corrente elétrica contínua (DC)/RF para formação do plasma, um filamento de tungstênio, um suporte circular em que se acomoda o substrato, um pulsador de alta tensão negativa e dois ímãs permanentes. A câmara apresenta formato cilíndrico, com 600 mm de diâmetro e 500 mm de altura. Ela possui entradas que permitem a formação do vácuo, a injeção de gases, a excitação do plasma, o controle de pressão e uma janela de vidro, como mostra a Fig. 1.

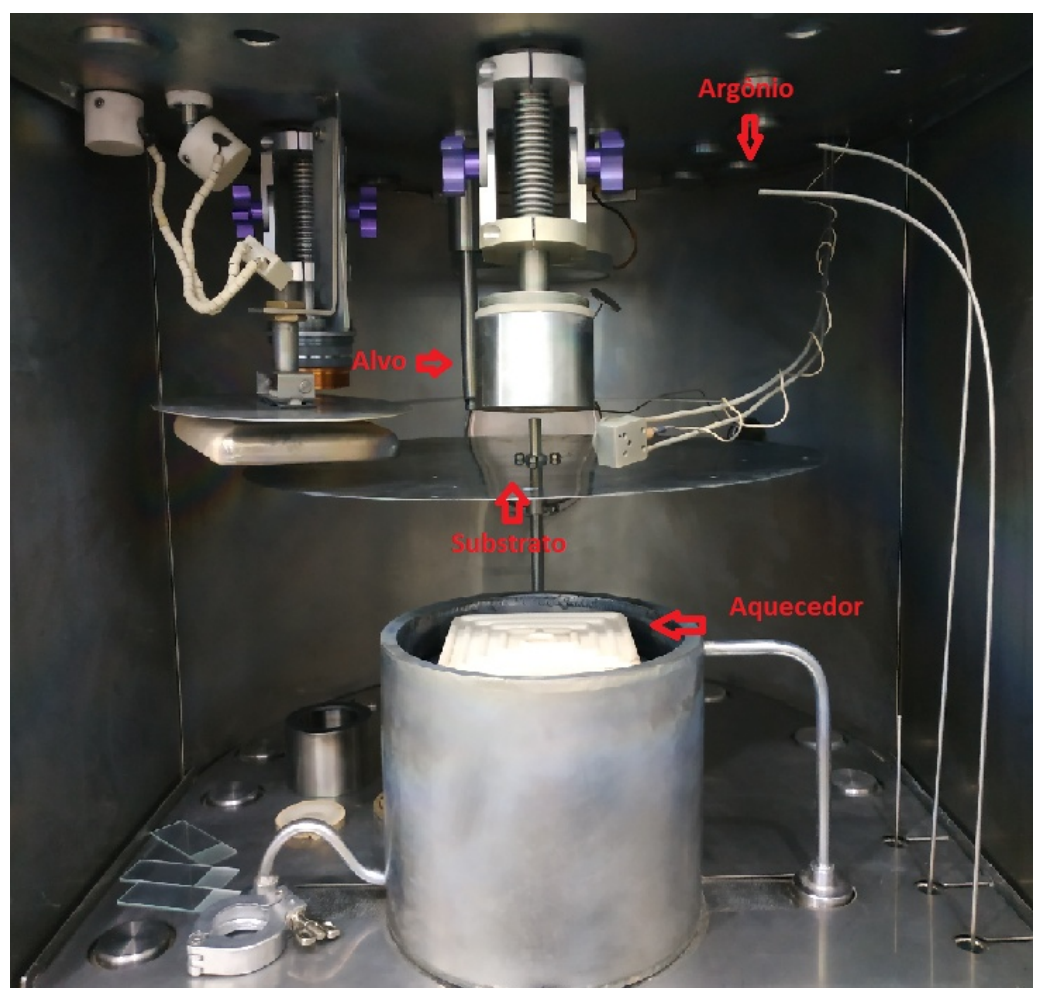

Figura 1: Interior de câmara de deposição.

No processo de deposição por sputtering, as propriedades dos filmes finos são influenciadas pelos parâmetros operacionais, como, por exemplo, a potência elétrica fornecida pela fonte de RF, a vazão de argônio (Ar), a temperatura dentro da câmara e no substrato, a pressão de processo e o tempo de deposição. Além disso, temos outros parâmetros importantes para o estudo, como a distância entre o alvo e o substrato e a pressão de trabalho. 
O plasma formado na evaporadora caracteriza-se como um de acoplamento capacitivo, em que o plasma é iniciado e sustentado por um oscilante campo elétrico (RF) em uma região entre dois eletrodos. A densidade do plasma é calculada com base em alguns parâmetros inseridos no modelo, como: geometria, dimensão e simetria; composição do gás; pressão; potência RF; e coeficientes de emissão secundária.

Este trabalho foi desenvolvido com o objetivo de simular o que ocorre dentro da câmara de deposição de filmes finos da evaporadora Prest Vácuo, em que foi realizada a deposição por sputtering RF em trabalhos experimentais já executados no laboratório de filmes finos da Universidade Estadual de Santa Cruz (UESC). O trabalho feito no laboratório consiste na deposição de filmes finos de óxido de zinco e alumínio ${ }^{3}$. O estabelecimento de uma rotina de simulação dos fenômenos físicos do que ocorre dentro da câmara em uma área limitada de interesse, ou seja, próximo aos eletrodos, focando em distribuição de densidade e temperatura eletrônica, na corrente e tensão e no potencial elétrico, tem como propósitos reconhecer o comportamento desse plasma e avaliar alguns parâmetros de deposição que podem influenciar na melhoria da produção de filmes finos.

\section{MATERIAIS E MÉTODOS}

Para a visualização da distribuição espacial da densidade do plasma na região de interesse próxima aos eletrodos, simulamos um modelo em duas dimensões (2D). É necessário determinar as variáveis como dimensão, geometria, modelo e parâmetros alinhados com os mesmos parâmetros de deposição dos estudos experimentais para a comparação e validação desses resultados no modelo matemático.

As principais condições de contorno do modelo são a geometria, a dimensão e a simetria, a composição do gás, a pressão, a potência RF e os coeficientes de emissão.

No software de simulação, selecionamos o estudo em 2D axissimétrico, ou seja, com simetria cilíndrica e rotação no eixo z. O módulo plasma em tempo periódico (ptp) foi selecionado como o tipo de estudo para a resolução do problema nos domínios harmônico, periódico e estacionário. Inserimos as variáveis da Tab. 1. A geometria inclui um ponto para ajudar na formação da malha, para redução de número de graus de liberdade, criando uma geometria que reduz a demanda do tempo de computação e requisitos de memória.

Tabela 1: Parâmetros para as variáveis utilizadas na simulação em duas dimensões.

\begin{tabular}{ccc}
\hline Variável & Valor & Descrição \\
\hline $\mathrm{l}$ & $28 \mathrm{~mm}$ & Comprimento (distância entre os eletrodos) \\
\hline $\mathrm{R}$ & $80 \mathrm{~mm}$ & Raio do cátodo \\
\hline $\mathrm{R}_{\mathrm{a}}$ & $140 \mathrm{~mm}$ & Raio do ânodo \\
\hline $\mathrm{L}_{\mathrm{d}}$ & $3 \mathrm{~mm}$ & Comprimento material dielétrico em contato com o cátodo \\
\hline $\mathrm{P}_{\mathrm{abs}}$ & $1 \mathrm{~W}$ & Potência \\
\hline $\mathrm{f}_{0}$ & $13,56 \mathrm{MHz}$ & Frequência \\
\hline $\mathrm{P}_{0}$ & $13,332 \mathrm{~Pa}$ & Pressão do gás $(0,1$ Torr) \\
\hline $\mathrm{T}_{0}$ & $350 \mathrm{~K}$ & Temperatura \\
\hline
\end{tabular}

Assim, na geometria foram inseridos dois retângulos: o retângulo 1, com comprimento de $140 \mathrm{~mm}$ e altura de $28 \mathrm{~mm}$ na posição inicial de $0 \mathrm{~mm}$; e o retângulo 2, com $56 \mathrm{~mm}$ de comprimento e $30 \mathrm{~mm}$ de altura na posição de $88,28 \mathrm{~mm}$. Foram feitos um chanfro de $6 \mathrm{~mm}$ e dois filetes nas extremidades do chanfro com $3 \mathrm{~mm}$, e inseriu-se um ponto na posição $80 \mathrm{~mm}$ para identificar e gerar a malha, o que simplifica os cálculos. Adicionou-se entre os retângulos um segmento de reta para separar as áreas na malha em que, posteriormente, se realizou refinamento apenas na região de maior interação do plasma, como se observa na Fig. 2. 
(a)

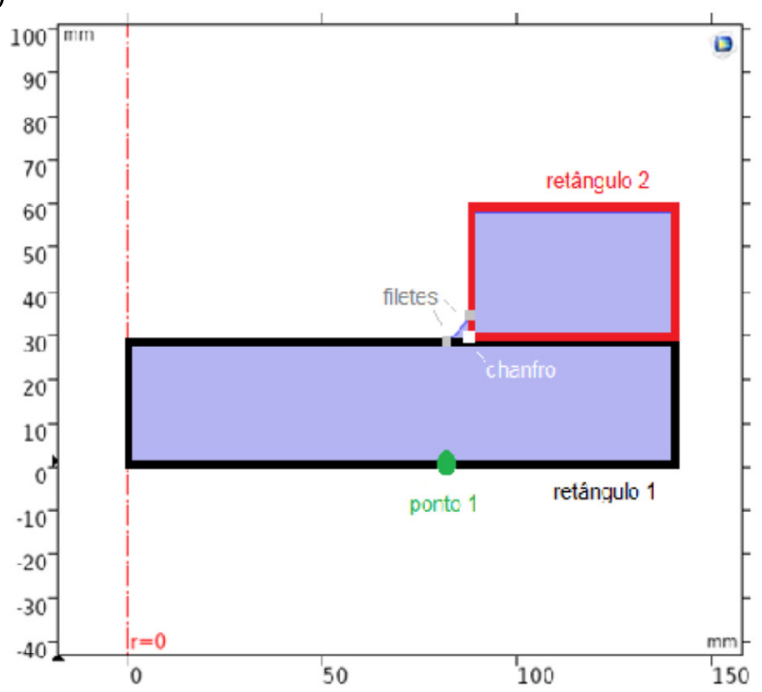

(b)

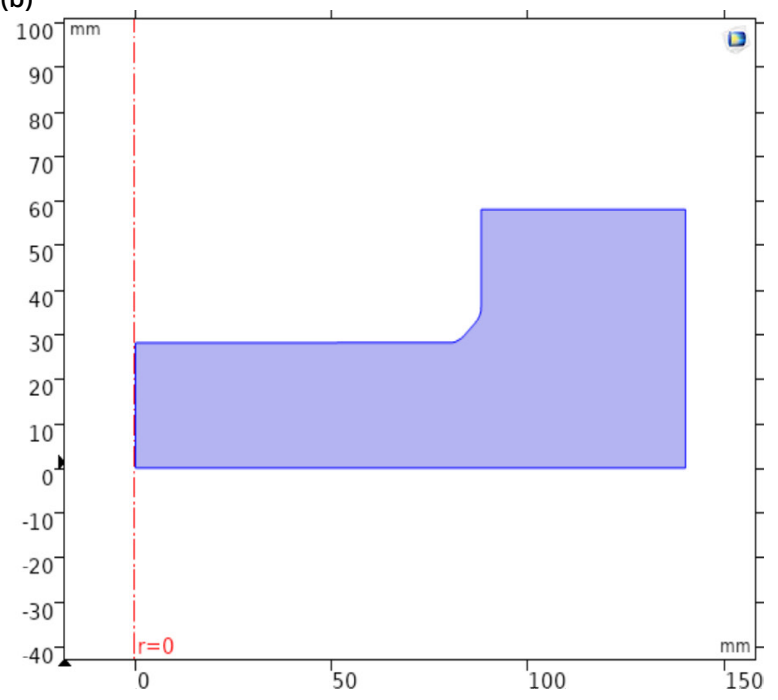

Figura 2: Geometria final em duas dimensões do modelo modelado no Comsol Multiphysics. (a) Um explicativo da geometria adicionado; (b) a geometria final.

O estudo do plasma no Comsol Multiphysics pelo método dos elementos finos (MEF) é realizado apenas em um sistema para a qual há colisão entre elétrons e íons. As colisões de elétrons são caracterizadas por seções transversais que precisam ser fornecidas pelo usuário. Como o argônio possui um esquema de reações simples, usamos uma química que descreve sete reações de volume envolvendo elétrons, íons atômicos e nível concentrado, representando os estados de argônio 4s.

Para a inserção do argônio no modelo, foi utilizado o arquivo de dados específicos disponível no banco de dados do software ${ }^{4}$. Considerou-se que o íon usa sua energia interna para extrair um elétron da parede com probabilidade de 0,07 e energia média de 5,8 V. O coeficiente de aderência foi igual a 0, e isso significa que entendemos que as perdas para a parede seriam toda a superfície, excluindo apenas o eixo de simetria, que foi rotacionado.

Assim, vamos adicionar uma malha especifica apenas na geometria de base, região em que se situam os eletrodos, e usar 30 elementos na dimensão extra que representam um período de RF, além de imputar a temperatura inicial $T_{0^{\prime}}$ a pressão do gás $p_{0}$ e as propriedades de densidade e energia dos elétrons no transporte. Especificamos a mobilidade em $3 \times 10^{5}\left(\mathrm{~cm}^{2} /(\mathrm{V} \cdot \mathrm{s}) / 0,1\right)$.

Outra condição de contorno adicionada foi a identificação do eixo de simetria em que ocorre a rotação da geometria. Definiu-se o alvo ou eletrodo (cátodo) como uma superfície metálica em que foram adicionadas a potência $P_{a b s}$ e a frequência $f_{0}$ e foi especificado o uso da fonte do tipo RF e DC com a opção DC self-bias. O material dielétrico e o terra estão localizados conforme a Fig. 3.

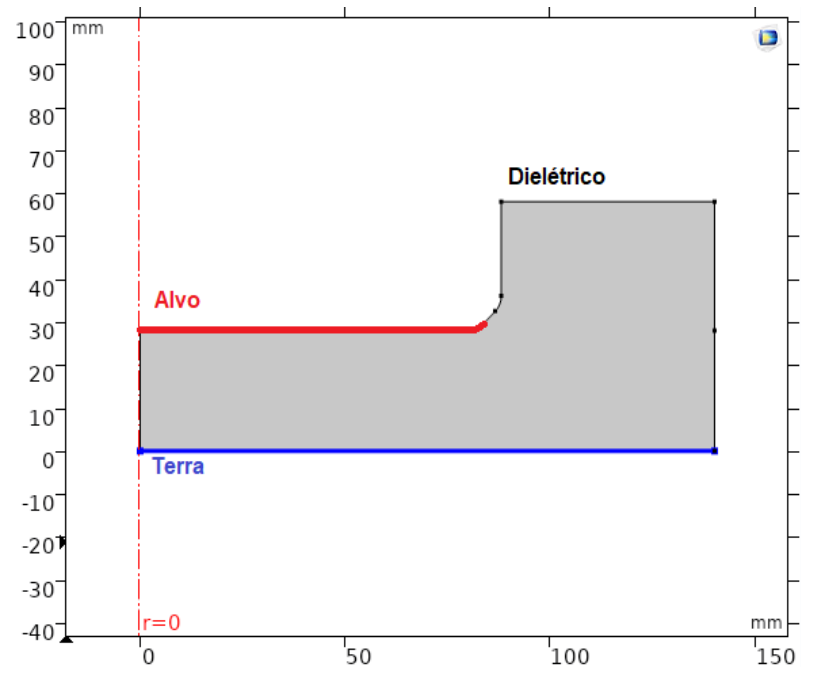

Figura 3: Geometria criada com a identificação de eletrodo cátodo (alvo), ânodo (terra) e material dielétrico. 
É muito mais estável configurar o eletrodo com uma potência fixa em vez de uma voltagem. Isso permite que os contatos e terminais sejam acionados diretamente com uma potência fixa, em vez de uma tensão fixa, o que aumenta a estabilidade numérica.

Para gerar a malha e minimizar o número de graus de liberdade, foi criada uma malha na direção axial e radial em que ocorre a descarga, região 1, e usamos uma malha mais grossa na região 2 (Fig. 4). Então, a geometria foi mapeada, e definiu-se uma malha para cada região. Na malha perto dos eletrodos (região 1), na qual a energia é absorvida, executamos uma distribuição de 600 elementos e refinamento apenas nessa região em que ocorre a descarga e mostra grandes gradientes. Na segunda região (região 2) foi utilizada uma malha triangular livre. Finalizado o processo, realizou-se a simulação, que teve duração total de 45 minutos.

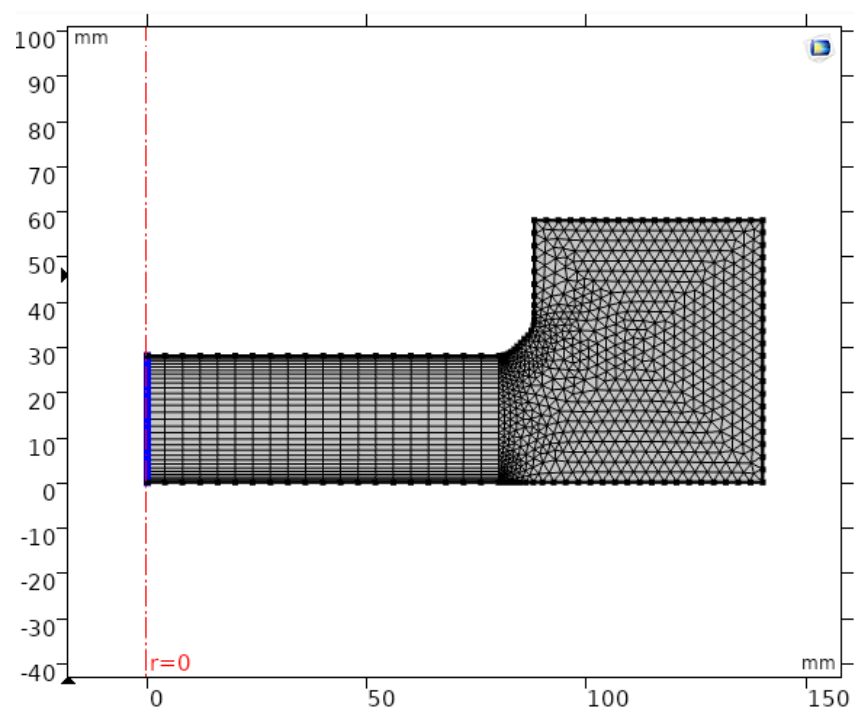

Figura 4: Formação da malha com distribuição específica para cada região.

A UESC possui a licença de uso do software Comsol Multiphysics, o qual está disponível para acesso no Núcleo de Biologia Computacional e Gestão de Informações Biotecnológicas. Nesse modelo, para a execução das simulações, foi necessária a utilização de um computador com 24 GB de memória RAM.

\section{RESULTADOS E DISCUSSÃO}

A complexa física do plasma com acoplamento capacitivo apresenta desafios para a realização de simulações numéricas em razão da existência de uma bainha de plasma, do comportamento dinâmico do plasma e do grande número de ciclos de RF necessários para atingir um estado estacionário periódico. Deposição de energia no plasma é altamente não linear, e o campo elétrico na bainha do plasma pode levar a instabilidades numéricas.

Os resultados apresentados são para $1 \mathrm{~W}$ de potência absorvida pelo plasma no modelo. Na Fig. 5, visualiza-se a densidade dos elétrons. Nesse gráfico podemos observar a baixa densidade na região próxima aos eletrodos, comportamento já esperado. Isso se dá por causa da blindagem de Debye, por intermédio da qual as partículas do plasma tendem a neutralizar qualquer carga elétrica que seja nele inserida. A distância característica dessa blindagem, chamada de comprimento de Debye, faz com que os elétrons e íons sejam confinados pelo potencial dessas regiões. É possível calcular o comprimento da bainha usando as Eqs. 1 e 2 e a densidade dos elétrons pela Eq. 3.

$$
\begin{gathered}
\lambda_{D e}=\sqrt{\frac{\epsilon_{0} T_{e}}{e n_{0}}} \\
\lambda_{D e}=\sqrt{\frac{\epsilon_{0} T_{e}}{e n_{0}}}
\end{gathered}
$$




$$
n_{0}=\frac{P_{a b s}}{e u_{B} A_{e f f} \varepsilon T}
$$

em que: $\lambda_{\text {De }}=$ comprimento de Debye $(\mathrm{m}) ; \epsilon_{0}=$ Permissividade no vácuo $\left(8,854 \times 10^{-12} \mathrm{~F} / \mathrm{m}\right)$; $T_{e}=$ Temperatura do elétron (eV); e = Carga elétrica do elétron $\left(1,602 \times 10^{-19} \mathrm{C}\right) ; \mathrm{n}_{0}=$ Densidade do plasma $\left(\mathrm{m}^{-3}\right) ; \mathrm{n}_{\mathrm{e}}=$ Densidade do elétron $\left(\mathrm{m}^{-3}\right) ; \mathrm{P}_{\mathrm{abs}}=$ Potência $(\mathrm{W}) ; \mathrm{u}_{\mathrm{B}}=$ Velocidade de Bohm $(\mathrm{m} / \mathrm{s}) ; \mathrm{A}_{\text {eff }}=$ Área efetiva do plasma $\left(\mathrm{m}^{2}\right) ; \varepsilon_{\mathrm{T}}=$ Energia total equivalente da tensão $(V)$.

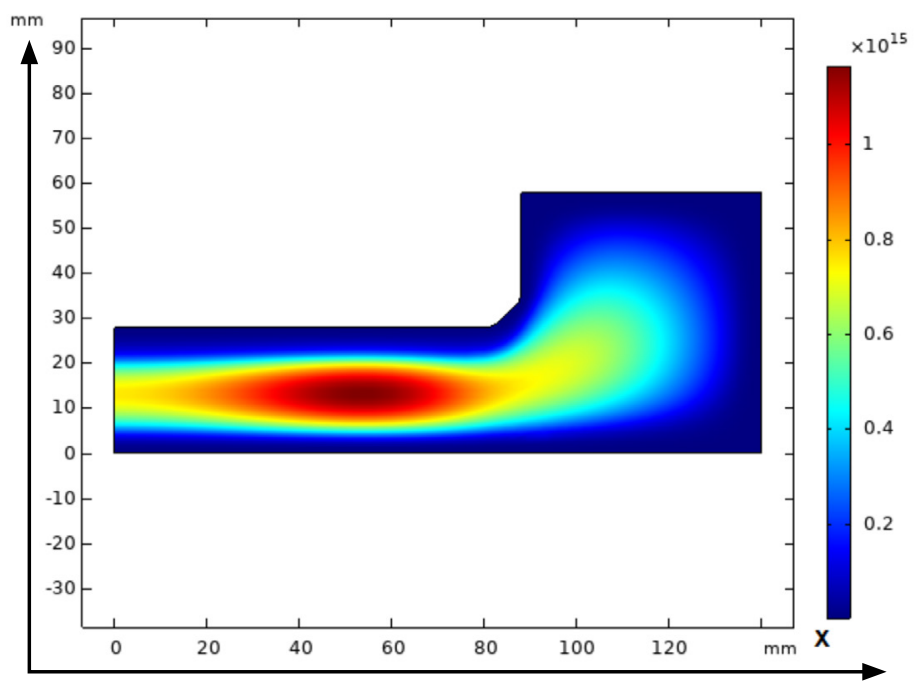

Figura 5: Densidade dos elétrons para visualização da distribuição em duas dimensões.

Na Tab. 2 exibimos os resultados obtidos da $T_{e}$ e $n_{e}$ pelo Comsol Multiphysics e, com base nesses resultados, calculamos o comprimento de Debye.

Tabela 2: Variáveis usadas para o cálculo do comprimento de Debye e o resultado encontrado para o modelo em uma dimensão.

\begin{tabular}{ccc}
\hline Variável & Descrição & Comsol \\
\hline$T_{e}$ & Temperatura do elétron & $11,9 \mathrm{eV}$ \\
\hline $\mathrm{n}_{\mathrm{e}}$ & Densidade do elétron & $1,55 \times 10^{15} \mathrm{~m}^{-3}$ \\
\hline$\lambda_{\mathrm{De}}$ & Comprimento de Debye & $0,651 \mathrm{~mm}$ \\
\hline
\end{tabular}

Esse resultado mostra que a bainha demonstrada no gráfico está em concordância com a Eq. (2), sendo um dos critérios de existência do plasma a quase neutralidade. Ou seja, o campo elétrico perturbativo que surge no interior de um plasma deve anular-se (blindagem de Debye) para distâncias maiores que $\lambda_{\text {De }}$. Para validação dos resultados apresentados na simulação (Fig. 6), determinamos a densidade de elétrons no centro do plasma (1/ $\left.\mathrm{m}^{3}\right)$ com base no modelo analítico ${ }^{5}$.

O resultado encontrado para a densidade do plasma foi igual a 2,05×1015 $\mathrm{m}^{-3}$, próximo do alcançado na simulação, de 1,55×1015 $\mathrm{m}^{-3}$, considerando aproximações definidas pelo modelo simplificado proposto na referência. Embora a densidade eletrônica seja muito baixa em comparação aos valores encontrados na literatura, próxima a $10^{16} \mathrm{~m}^{-36}$, podemos afirmar que esse plasma possui as densidades eletrônicas em bom acordo com o modelo teórico. Esses parâmetros caracterizam o plasma formado nessa simulação como pouco energético, o que é justificado em razão da baixa potência entregue ao plasma (1 W).

Neste estudo, o objetivo foi obter uma solução a $100 \mathrm{~W}$ conforme a potência utilizada nos estudos experimentais da evaporadora. Para isso, começamos a simulação com 1 W na condição inicial para posteriormente atingir 100 W, sendo mais fácil começar com uma potência baixa e depois varrer a energia até que a potência desejada fosse atingida. Definir um incremento de potência máxima para o solucionador paramétrico torna a convergência mais suave à medida que a potência é aumentada. A Fig. 6 mostra a simulação da densidade com o estudo paramétrico variando a potência fornecida 
ao plasma de 1 a 100 W. Visualizando um aumento significativo da densidade, alcançam-se valores próximos ao desejado. Quando inserimos potências maiores que 100 W, as interações numéricas não convergem para uma solução.

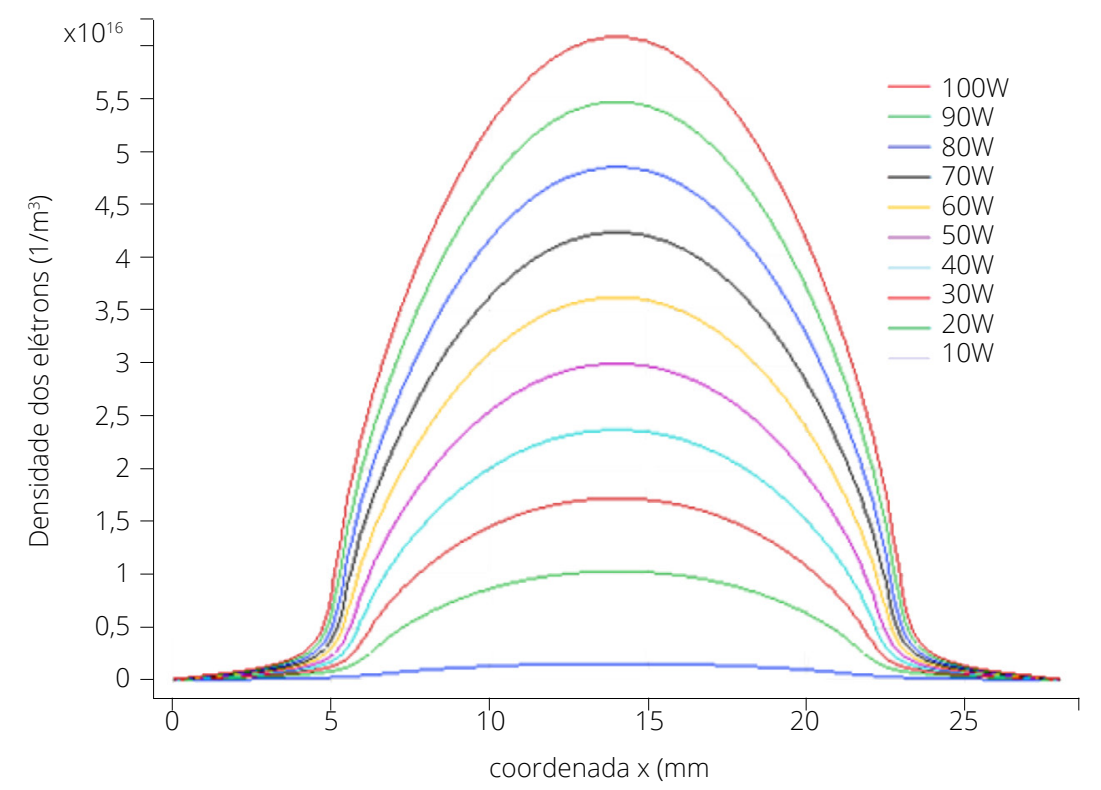

Figura 6: Estudo paramétrico da densidade do plasma. Aumentou-se a potência fornecida ao plasma de 1 a $100 \mathrm{~W}$ em modelo unidimensional a partir da distância entre os eletrodos (28 mm).

Podemos verificar na Fig. 7 a distribuição da densidade de elétron no plasma. Nessa região o plasma formado dentro da bainha não tem um comportamento simétrico considerando o eixo x (axial) nem o eixo y (radial). Com essa análise, executamos uma seleção nos eixos radial e axial levando em conta a posição, dentro da câmara, da amostra e dos eletrodos. É necessário salientar que é nessa posição (no centro), dentro da câmara do reator, que acomodamos o substrato durante o processo de deposição dos filmes finos. Se observarmos o eixo de simetria na simulação, podemos entender por que na produção desses filmes o crescimento apresenta valores menores de espessura nas bordas das amostras. Podemos observar o valor baixo no centro da câmara, quando o comparamos com os resultados na dimensão 1,55×1015 $\mathrm{m}^{-3}$. Pela Fig. 5, as maiores densidades não estão nessa posição. Para isso, geramos os gráficos novamente e escolhemos os pontos y $=14 \mathrm{~mm}$ para o eixo axial e $\mathrm{x}=55 \mathrm{~mm}$ para o eixo radial, que apresentam a maior densidade de elétrons, conforme a Fig. 8.

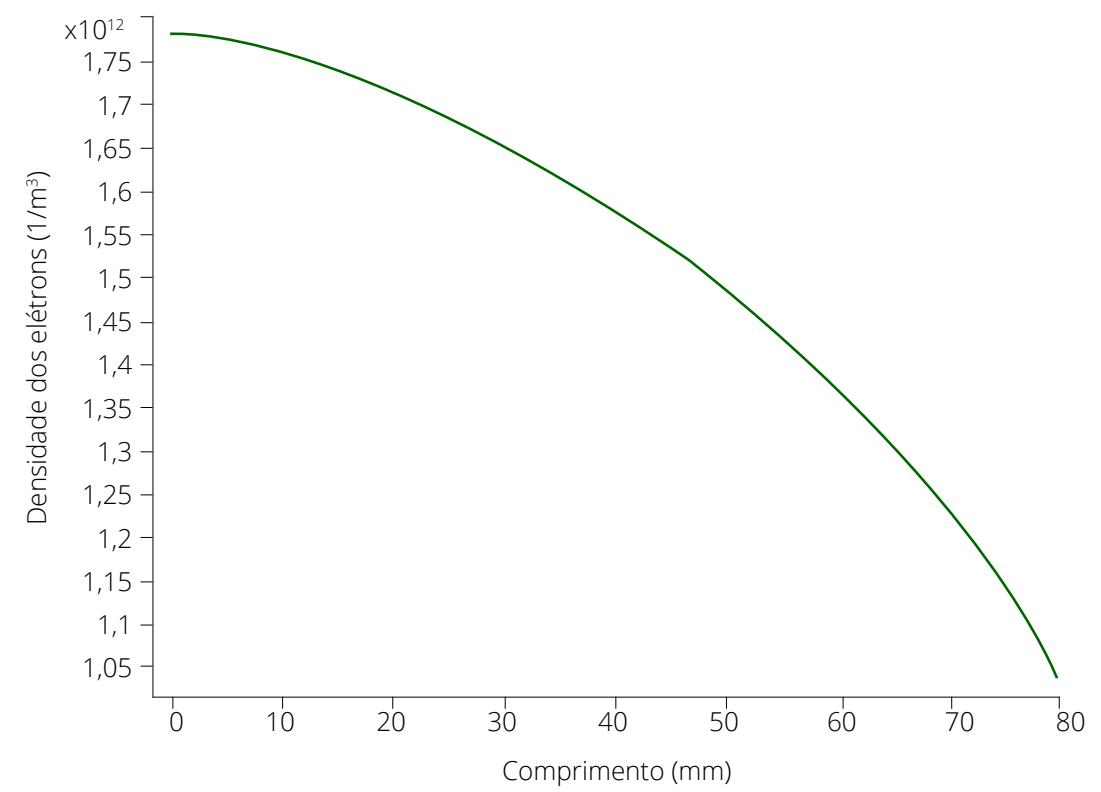

Figura 7: Distribuição da densidade de elétrons no eixo axial, com raio do alvo igual a 80 mm. 


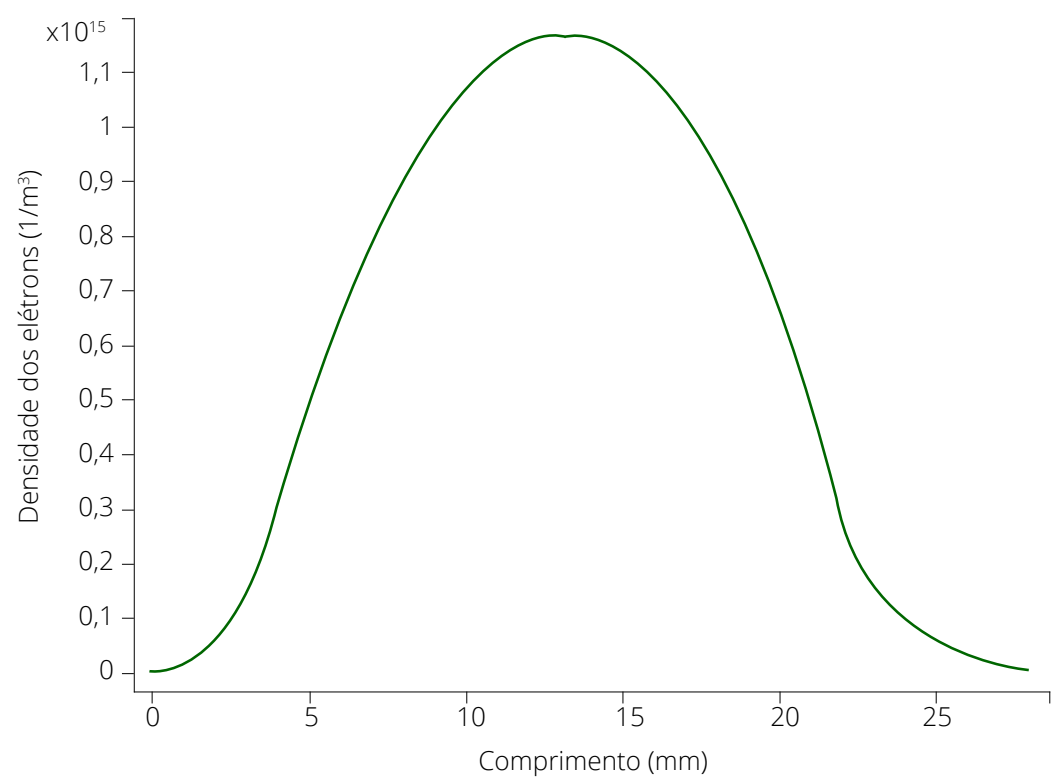

Figura 8: Distribuição da densidade de elétrons no eixo radial, com distância de 25 mm entre alvo e substrato no ponto em que x = 55 .

Essa distribuição da densidade é um resultado importante quando a relacionamos com o que acontece durante o processo de deposição. Essa formação mostra o local de maior densidade de elétrons e, com isso, podemos associá-la à maior área de incidência dos elétrons no alvo. A geometria representada na Fig. 5 pode ser vinculada à erosão que ocorre no alvo após sucessivas deposições de filmes finos (Fig. 9).

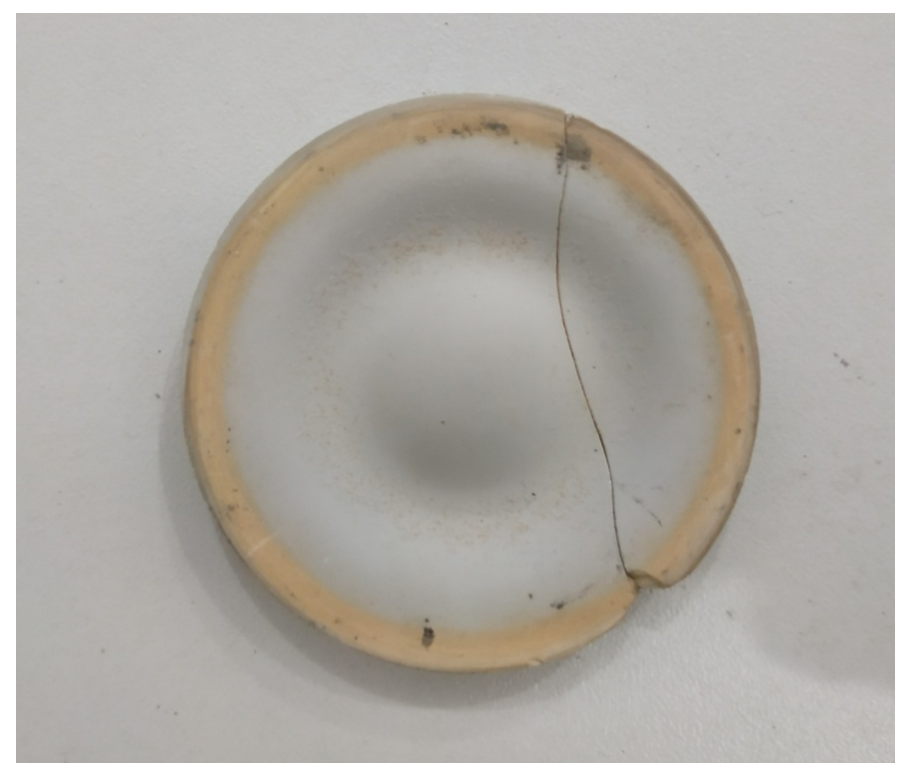

Figura 9: Alvo (zinco) utilizado para a deposição de filmes finos de óxido de zinco.

\section{CONCLUSÕES}

O conhecimento do comportamento do plasma permite avaliar a influência de diferentes parâmetros para a deposição de filmes finos, como a densidade dos elétrons, a temperatura, o potencial elétrico, a corrente e a tensão, além da distribuição espacial do plasma, o que deve favorecer a deposição de filmes finos em uma região mais próxima das amostras. Neste trabalho, apresentamos um modelo bidimensional para aplicação de plasma pelo método de simulação por elementos finitos. 
Na abordagem desenvolvida foi verificada a presença da distância de Debye, um critério importante que caracteriza o comportamento em grupo do plasma e a formação da bainha. A formação dessa blindagem diferencia o modelo de gás apenas ionizado e um plasma. É possível visualizar tal comportamento nesse modelo e a variação dessa formação da bainha entre os eletrodos, além de como a distância de Debye é menor se comparada ao tamanho total do plasma, demonstrando que as interações do interior do plasma são mais importantes do que aquelas que ocorrem nas bordas. A densidade de elétrons indica esse comportamento de quase neutralidade do plasma confirmando que a maior amplitude de densidade se encontra no centro do plasma. A compatibilidade dos resultados simulados com o modelo global para a densidade dos elétrons valida as equações contidas na simulação.

Apesar da desproporcionalidade em relação à geometria do equipamento (diâmetro do alvo e distância dos eletrodos), os resultados da simulação, considerando os eixos axial e radial, apresentam padrões compatíveis com as medidas que idealmente deveriam ser utilizadas.

Para variações na potência de 1 a 100 W, houve aumento na densidade de elétrons gerando um plasma mais energético, entretanto o modelo alcançou o limite de $100 \mathrm{~W}$, não permitindo o incremento de maiores potências, mas foi possível verificar que com o aumento da potência ocorre o aumento da densidade dos elétrons, apontando crescimento linear.

Ainda, observamos que ao longo dos eixos axial e radial a distribuição da densidade de elétrons não se apresenta de forma simétrica. Realizamos um estudo para a identificação da região em que ocorre a maior densidade para melhor posicionamento das amostras. Nesse sentido, espera-se que nessa posição ocorram melhores taxas de deposição, distribuição e redução de falhas nas bordas do substrato.

Finalmente, com base nos resultados deste trabalho, foi simulado um plasma com características próximas da câmara de deposição do Laboratório de Filmes Finos. Além disso, os parâmetros de deposição de entrada mostraram concordância com os resultados paramétricos apresentados (potência e pressão), confirmando os resultados experimentais de deposição.

A simulação da máquina de deposição possibilitou identificar a falha apresentada no consumo do alvo, por meio da assimetria da formação do plasma pelo posicionamento nos eixos axial e radial e cujo resultado encontrado diverge do esperado inicialmente, que seria um desgaste maior no centro do disco e a formação do plasma e densidade de elétron maior no centro do eixo de simetria.

\section{REFERENCES}

1. Smith DL. Thin film deposition: principle and practice. Nova York: McGraw Hill; 1995.

2. Vahedi V, Birdsall CK, Lieberman MA, Dipeso G, Rognlien TD. Verification of frequency scaling laws for capacitive radio-frequency discharges using two-dimensional simulations. Phys Fluids B. 1993;5(7):2719-29. https://doi. $\operatorname{org} / 10.1063 / 1.860711$

3. Viana W, Pisani MB, Segundo GSA, Correia NS. Filmes finos de óxido de zinco depositados por RF-magnetron sputtering na presença de oxigênio. Rev Bras Aplicação Vácuo. 2017;36(3):114-7. https://doi.org/10.17563/rbav. v36i3.1053

4. Comsol. Plasma Module User's Guide. Comsol® Multiphysics; 2017 [Internet]. [acessado em ago. 2019]. Disponível em: https://doc.comsol.com/5.3/doc/com.comsol.help.plasma/PlasmaModuleUsersGuide.pdf

5. Lieberman MA, Lichtenberg AJ. Principles of plasma discharges and materials processing. 2. ed. Hoboken: WileyInterscience; 2005.

6. Boyd TJM, Sanderson Jj. The physics of plasmas. Cambridge: Cambridge University Press; 2003. 